\title{
Aspectos sobre la evaluación, fortalezas y áreas de oportunidad del programa nacional de convivencia escolar en una escuela de Yucatán
}

\author{
Shirlei Elizabeth Gazca Vazquez \\ shirleigazca92@gmail.com \\ Ángel Martín Aguilar Riveroll \\ aguilarr@correo.uady.mx \\ Universidad Autónoma de Yucatán \\ Mérida, México
}

\section{RESUMEN}

El estudio de la violencia escolar en México se ha convertido en un campo de interés para la investigación. Varios países ya cuentan con programas cuya intención es promover el respeto y la convivencia. En México, existe el Programa Nacional de Convivencia Escolar (PNCE). Actualmente hay poca evidencia sobre su implementación. Vale la pena sumar esfuerzos para realizar diagnósticos en distintos estados que notifiquen sobre cómo se lleva a cabo. El objetivo de este trabajo es analizar la percepción que tienen un representante estatal del programa, maestros y el director de una escuela de Yucatán, sobre la retroalimentación recibida, opiniones, aciertos y áreas de oportunidad del programa. Se optó por un diseño cualitativo con el que, a partir de entrevistas semiestructuradas, se encontraron respuestas que ponen de manifiesto la relevancia de la formalización de los procesos de evaluación y retroalimentación del programa; la importancia de visibilizar temas crítcos que acongojan al país, y que son tratados por el PNCE, la necesidad de estrechar las relaciones entre la administración del programa y los miembros de la escuela, en función de crear ambientes que contribuyan a la convivencia en los centros educativos de México.

Palabras clave: programa escolar; convivencia escolar; violencia escolar. 


\title{
Evaluation, strengths and weaknesses of the national school coexistence program in a school in Yucatán
}

\begin{abstract}
The study of school violence in Mexico has become an area of research interest. Nowadays many countries have school programs that intend to promote respect and coexistence. In Mexico, there is the National School Coexistence Program (PNCE), currently there is only little evidence about its implementation. It is worth to make efforts to carry out diagnoses in different states that report how it is carried out. Therefore, our goal is to analyze the perception that a state representative of the program, teachers, and the director of a school in Yucatan have about the feedback received, opinions, strengths and weaknesses of the program. We designed a qualitative study. For data collection, we carried out semi-structured interviews. The results show the relevance for the formalization of the evaluations and feedback processes; the importance of making visible critical issues that afflict the country, and that are addressed by the program, it also showed the need to strengthen relations between the administration of the program and the members of the school.
\end{abstract}

Key words: school program; coexistence; national program; school violence

Artículo recibido: 10. Junio. 2021 Aceptado para publicación: 16. Julio. 2021 Correspondencia: shirleigazca92@gmail.com Conflictos de Interés: Ninguna que declarar 


\section{INTRODUCCIÓN}

Desde finales de los noventa y comienzos del siglo XXI la atención por estudiar temas relacionados con la violencia y la convivencia escolar adquirió una orientación global (Carro Olvera, 2018 y Martínez y Góngora, 2011). La promoción e inclusión de la convivencia como uno de los cuatro pilares fundamentales para la educación del siglo XXI en Latinoamérica quedó plasmada desde 1996 en el informe de Delors por la UNESCO. En México, el estudio de la violencia escolar se ha convertido en un campo de investigación, en el que ha permeado el interés por conocer las causas, a los actores que intervienen, las implicaciones y los modos en los que se manifiesta (Saucedo Ramos y Guzmán Gómez, 2018). Actualmente, varios países ya cuentan con programas cuya intención es promover el respeto y la convivencia en los centros educativos.

Tal es el caso de México, con el Programa Nacional de Convivencia Escolar (PNCE), puesto en marcha en el ciclo escolar 2016-2017, con el objetivo de favorecer el establecimiento de ambientes de convivencia armónica y pacifica y para prevenir situaciones de acoso escolar en escuelas publicas de educación básica; así como de impulsar ambientes de relación y de convivencia favorables para la enseñanza y el aprendizaje en las escuelas (Secretaría de Educación Pública, 2016). El programa es de carácter formativo y preventivo, cada entidad federativa del país cuenta con equipos técnicos a quienes se les capacita con el fin de promover la intervención pedagógica.

El PNCE (Secretaría de Educación Pública, 2016), propone una estrategia de capacitación en varias etapas con la finalidad de garantizar que la capacitación e información lleguen a supervisores, directivos y colectivos docentes de las escuelas participantes. En una primera etapa el equipo de asesores de la Dirección del PNCE capacita al equipo técnico de la Coordinación Estatal del Programa, este, con la asesoría y acompañamiento del personal de la Dirección del PNCE, replica la capacitación a los jefes de sector y asesores técnicos pedagógicos y supervisores; posteriormente, estas figuras educativas, con la asesoría y acompañamiento del equipo técnico estatal, son los responsables de capacitar a directores y docentes de las escuelas. Las capacitaciones otorgadas permanecen en forma de asesoría y acompañamiento durante el proceso de implementación del PNCE. Con esto, se busca atender las necesidades de información, resolver dudas y revisar las estrategias didácticas para mejorar las actividades propuestas en los materiales educativos 
que el programa otorga. Esta asesoría y acompañamiento se puede ofrecer en los Consejos Técnicos Escolares (CTE).

En lo que respecta al seguimiento del programa en las escuelas se establece una estrategia en la que, a través de una evaluación, se obtengan datos referentes sobre clima y convivencia en las escuelas donde se implementa el PNCE. Los instrumentos aplicados tienen la función de otorgar resultados sobre aspectos clave del programa, en temas de convivencia y habilidades sociales y emocionales, así como de temas relacionados al objetivo y otros factores relevantes del PNCE. Al ser aplicados mediante un sistema en línea se obtienen resultados de cada escuela de forma inmediata. Saucedo Ramos y Guzmán Gómez (2018), por su parte, advierten que una característica en común de los programas desarrollados para erradicar la violencia en el país es la falta de evaluación de su impacto y la manera como fueron recibidos por la comunidad educativa. En cuanto al PNCE, dentro de las principales recomendaciones de la evaluación 2017-2018 del CONEVAL, se sugiere considerar la posibilidad de llevar a cabo estudios "para conocer los procesos a nivel escolar de recepción, aprobación y uso de los componentes a nivel escuela y aula" (Secretaría de Educación Pública, 2017, p.59) pues existe una debilidad identificada en la evaluación debido a la falta de un indicador especializado para los resultados de la intervención y no sólo de cobertura.

En México, las evaluaciones sistemáticas a los programas que se implementan son un proceso joven, si bien en las últimas dos décadas ha sido posible vislumbrar avances en estos procesos (Conde-Bonfil, 2017) aún no se han logrado sistematizar.

En cuanto al PNCE, actualmente es posible encontrar unos primeros trabajos (ChaparroCaso-López, Mora-Osuna, y Medrano-Gallegos, 2018 y Rivero Espinosa, 2017) por realizar revisiones sobre cómo se lleva a cabo el programa en una entidad federativa específica. Es por lo anterior que vale la pena sumar esfuerzos para realizar análisis y diagnósticos de la implementación del PNCE que den cuenta de la forma en la que se lleva a cabo. Por ello, el objetivo de este trabajo es analizar la percepción que tienen un representante estatal del programa, un grupo de maestros y el director de una escuela de Yucatán incorporada al PNCE, sobre la retroalimentación recibida, opiniones, aciertos y áreas de oportunidad del programa. 


\section{MÉTODO}

Se escogió un diseño cualitativo ya que este permite indagar sobre el punto de vista de individuos de un contexto determinado (Creswell, 2009). Para la recolección de datos se realizaron entrevistas semiestructuradas debido a la posibilidad que ofrece de profundizar en el tema, las preguntas fueron revisadas por un grupo de jueces expertos. Se diseñaron tres guías de entrevista para cada clasificación de los sujetos de estudio. La guía final consta de 12 preguntas para el representante, 16 para el director y 14 para los maestros. Las entrevistas completas, fueron diseñadas para un trabajo de tesis académica, contienen preguntas sobre los objetivos específicos del PNCE, preguntas basadas en la evaluación del proceso según Rossi, Lipsey, y Freeman (2004) y sobre generalidades, aciertos y áreas de oportunidad del PNCE, de estas últimas se desprenden los resultados mostrados en este trabajo. Las entrevistas se realizaron a través de videollamadas o llamadas telefónicas según la preferencia de los entrevistados, esto con la finalidad de procurar el distanciamiento social debido a la pandemia por Covid-19. Tuvieron una duración máxima de una hora; las conversaciones fueron grabadas con previa autorización de los participantes; se realizaron las transcripciones de cada una y se realizó una codificación y categorización para el posterior análisis del contenido de las entrevistas.

En Yucatán existen 1193 escuelas inscritas al PNCE, de las cuales 507 son de educación primaria y se benefician 38,750 alumnos, 4370 docentes y 507 directores (Gobierno Estatal 2018-2024, 2018). Se realizó una selección por conveniencia de una escuela primaria la cual concedió los permisos necesarios para la recolección de datos. Se trata de una escuela vespertina de la ciudad de Mérida ubicada en una zona considerada con altos niveles de violencia. Cuenta con dos salones por año escolar y tienen un promedio de 26 a 30 alumnos por aula, con una matrícula total de 300 estudiantes. Los participantes de dicha escuela estuvieron conformados por el director y maestros de quinto y sexto que accedieron de manera libre y voluntaria a participar, en total dos maestros de sexto, uno de quinto y el director de la escuela. En el caso de la autoridad del PNCE, se solicitó la participación por medio de una carta y con el envío del protocolo del trabajo.

\section{RESULTADOS}

El representante del PNCE explicó cómo se llevan a cabo las evaluaciones al programa: 
Cada año se hace una evaluación en línea. Las escuelas nos califican a nosotros como programa. En algún momento, al finalizar el curso escolar, desde México, se manda un vínculo y las escuelas participantes se meten y califican el programa. Nos ha ido bien...bueno no estoy diciendo que sea perfecto...todo es perfectible, siempre hay una forma de mejorar, por eso agradezco todos los comentarios que los directores $y$ directoras me quieran hacer o le quieran hacer a los compañeros en las capacitaciones... (Representante).

Como sucede con cualquier modalidad de evaluación se pueden atravesar diversas situaciones que puedan poner en duda las respuestas obtenidas. Si bien las evaluaciones en línea tienen ventajas como el control, difusión y velocidad de procesamiento de la información, también tiene la desventaja de poder cambiar respuestas una vez enviado el formulario, como la situación sucedida, durante la administración previa, en la escuela y que es referida por uno de los maestros:

Recuerdo que, a medio curso, nos mandaron una encuesta que nosotros teníamos que contestar acerca del programa y había una pregunta, que recuerdo muy bien, sobre si a mí me habían dado la capacitación. Y yo puse que no, y así como yo puse que no, varios maestros también lo pusieron... La directora de ese momento nos mandó a llamar a la dirección, e hizo que borráramos lo que habíamos puesto y que pusiéramos que sí, que ella nos había dado la capacitación... (Maestro sexto grado).

Los informantes también destacaron sobre la importancia del seguimiento al PNCE ... sé que hay escuelitas en las que existe este programa, pero sólo llegan los libros y así como llegan se reparten y ya. Entonces pienso que sí se le debe dar seguimiento sin querer fiscalizar a nadie porque a veces no es por no querer hacerlo o no querer terminarlo sino por el factor tiempo... (Maestro quinto grado).

Sobre este seguimiento a las escuelas inscritas al PNCE, el representante menciona que no hay un protocolo formal. Refiere que personalmente ha tratado de visitar algunas escuelas y platicar con los directores con la finalidad de recibir cierta retroalimentación 
... yo en lo personal, trato de visitar unas treinta o cuarenta escuelas al año, pero mil ciento noventa y dos escuelas sería imposible la sola idea de visitarlas todas; sin embargo, por ejemplo, el año pasado me fui donde no llega ni los cobradores de tiendas ${ }^{1}$. Me fui por San Felipe, Río Lagartos, nunca habían ido. Trato de hacer cuando menos diez minutos, preguntando ¿cómo está?; ¿cómo le va?; ¿dónde está el material? De esa manera se puede ver dónde está el material, si el material es adecuado o nos dicen sobre las actividades que les hayan gustado. Conversar con los directores para mí es muy importante (Representante).

También menciona que la dificultad de darle un seguimiento puntual a cada escuela recae en el alto número de escuelas inscritas y el pequeño número del equipo del PNCE.

Sobre la divulgación de la información recibida por parte del PNCE, el director refiere que es precisamente a los directores de cada escuela a quienes se les capacita y ellos se encargan de la transmisión de la información a los docentes, misma que se hace en los CTE:

...después de la capacitación que nos dan a nosotros, nos comparten la presentación, los materiales trabajados en ese día. Lo que se hizo para hacer llegar esta información a los docentes fue que en un CTE trabajamos el tema del Programa Nacional de Convivencia Escolar y unos temas relacionados con lo socioemocional que nos presentaron en esa capacitación ... de hecho, como en la zona todas las escuelitas están en el programa fue un acuerdo con el supervisor. El supervisor nos dijo qué íbamos a hacer después de la capacitación y entre todos se tomó el acuerdo trabajar el Programa Nacional por una hora (Director).

De acuerdo con los lineamientos del PNCE, una vez que en las escuelas se cuente con la capacitación y los materiales educativos, las escuelas deben de integrar las actividades del Programa en la planeación de la Ruta de Mejora y durante las sesiones del Consejo Técnico Escolar (CTE) para favorecer la accesibilidad de la información. La información referida por el director es también descrita por uno de los maestros, quien destaca la

\footnotetext{
${ }^{1}$ El representante se refirió de manera concreta a una tienda departamental cuyo nombre se prefirió omitir y reemplazar por un aspecto más general
} 
importancia de dar tiempo en los CTE para tratar temas socioemocionales, al respecto menciona:

Creo que lo benéfico de estos nuevos consejos técnicos es que sí le están dando peso a esta parte de convivencia escolar. Antes, te estoy hablando de hace cinco o cuatro años, sí se tocaban esos temas, pero no se puntualizaban, en cambio ahora sí. Hay un eje dentro del CTE donde sí nos hacen trabajar esa parte de convivencia... porque antes era parte del currículo oculto de ser docente, el cómo manejar la tranquilidad y la paz en tu comunidad, en cambio ahora se maneja de manera formal y escrita dentro de nuestras actividades como docentes (Maestro quinto grado).

Si bien se espera que las actividades del programa no saturen las académicas, existe interés por parte de los maestros por conocer protocolos de actuación ante situaciones de violencia escolar, actividades y temas que favorezcan al desarrollo de los estudiantes y la comunidad educativa.

....sí me gustaría que se le dedicara más tiempo, que se enriqueciera con más actividades, pero no actividades que saturen sino en las que se pueda desarrollar el niño, los padres de familia o incluso nosotros. Ese tipo de capacitaciones o de cómo conocer los protocolos de actuación (Maestro quinto grado)

Sobre el tiempo dedicado a las capacitaciones en las escuelas, el representante del PNCE asegura, bajo el conocimiento de la carga académica y de otros programas que tienen en las escuelas de educación básica, que se procura otorgar las capacitaciones en un día, durante tres o cuatro horas.

A continuación, se presentan las diversas fortalezas del programa identificadas por los informantes:

Tabla 1: Fortalezas del PNCE identificadas por los informantes

\begin{tabular}{cl}
\hline \multicolumn{1}{c}{ Informante } & \multicolumn{1}{c}{ Descripción } \\
\hline Maestro sexto grado & $\begin{array}{l}\text { Está enfocado a mejorar la convivencia e involucra a todos los } \\
\text { actores del sistema educativo }\end{array}$ \\
\hline
\end{tabular}




\begin{tabular}{|c|c|}
\hline Maestro quinto grado & $\begin{array}{l}\text { El mayor acierto es que se están interesando y dándole } \\
\text { visibilidad a un tema que antes estaba rezagado, ahora es muy } \\
\text { diferente a como era hace diez años. Se ha hecho parte del } \\
\text { programa de estudios. Es bueno tener el respaldo de un } \\
\text { programa }\end{array}$ \\
\hline Maestro 2 sexto grado & $\begin{array}{l}\text { Manejan contenidos convenientes, necesarios, lúdicos y } \\
\text { adecuados para los niños... }\end{array}$ \\
\hline Representante & $\begin{array}{l}\text {...aborda problemas puntuales que están lacerando a nuestro } \\
\text { país como son la violencia y la desigualdad de género. Creo } \\
\text { que, desde donde yo lo miro, los materiales, al abordar } \\
\text { problemas torales y lacerantes como la violencia de género y el } \\
\text { acoso escolar son fundamentales... }\end{array}$ \\
\hline
\end{tabular}

Elaboración propia

Entre las áreas de oportunidad referidas por los docentes, destacan la necesidad de que las capacitaciones lleguen a todos los maestros, alumnos e incluso padres de familia, de manera que se puedan aprovechar mejor los materiales otorgados. También se enfatiza en la necesidad de tener más tiempo de capacitaciones, así como mayor intervención de los encargados del programa. Por su parte, el representante también coincide con la importancia de proveer capacitaciones pertinentes. Además, asegura que el mayor reto es el acceder a las escuelas:

El llegar a las escuelas, para mí es el mayor reto. Y la mayor tristeza es no llegar a todas las escuelas; pero pues es imposible... no es solamente una cuestión económica, que ahora por los recortes sí es una parte importante, pero el mayor reto es llegar al mayor número de escuelas y por otro lado dar una capacitación pertinente, eficiente y eficaz, con temáticas que el maestro realmente sienta que les sirvan, no solamente para llenar un requisito...(Representante).

\section{DISCUSIÓN Y CONCLUSIONES}

Cada uno de los participantes describió algunas sugerencias que, desde su perspectiva, pueden contribuir a la mejora del programa. Por parte de los maestros y el director la 
sugerencia general es en relación con el tiempo dedicado a las capacitaciones; el acompañamiento del personal; los vínculos entre los miembros del programa y la escuela:

Que existieran personas que acompañen a los maestros, o que esas estuvieran integradas a nuestra comunidad. Que no sea una sola visita, sino que pudieran estar ahí en la escuelita (Maestro quinto grado).

Mejoraría la parte de ser más cercano con las escuelas ... igual que se logre llevar a algunos responsables a sensibilizar tanto a nosotros como a los padres de familia. Creo que es la comunidad que más requiere de este trabajo (Maestro 2 sexto grado).

Cambiaría el acompañamiento a las escuelas... pienso que es necesario guiarnos un poco más, es cansado entre tantos pendientes y cosas qué hacer en la escuela, pero sí considero que es necesario... (Director).

La sugerencia por parte del representante se relaciona también con los vínculos con las escuelas; con la necesidad de personal para poder tener mayor cobertura pues afirma que se tiene mucha demanda:

Pondría un turno vespertino, lamentablemente tenemos demanda, pondría más personal, daría el material por alumno y fortalecería las relaciones con los directores...Teniendo un turno que se abocara a las escuelas vespertinas se podría tener más cobertura, de nosotros, no del programa pues digamos que un $30 \%$ de las escuelas de nivel básico está en el PNCE (Representante).

Sobre las capacitaciones y la retroalimentación recibida por parte del programa, los testimonios son consistentes con lo referido por Chaparro-Caso-López et al. (2018), en donde los participantes refirieron lo siguiente: "Está muy bien todo lo que nos presentan, pero pienso que deberían apoyarnos con darnos seguimiento cuando llevemos a cabo el programa, para que nos guíen si vamos bien o mal (...) porque es muy poco el tiempo que tuvimos de capacitación y nos quedamos con algunas dudas" (p.10). Otros autores (Cerezo-Ramírez y Sánchez-Lacasa, 2013; Piña-Miramar, Tron-Álvarez, y BravoGonzález, 2014 y Rincón-Vargas, 2017), también coinciden con que es necesaria la capacitación a los docentes para llevar a cabo de manera adecuada los programas de convivencia. 
En cuanto a las evaluaciones, si bien es de reconocer los esfuerzos por parte de la coordinación por realizar preguntas informales y tener una escucha activa sobre las necesidades de las escuelas, incluso las más arraigadas, la situación general con el PNCE concuerda con la constante referida por Saucedo Ramos y Guzmán Gómez (2018) sobre la falta de evaluación a los programas en el país; si bien como mencionó Conde-Bonfil (2017) esto es aún un proceso joven, para conocer sobre los aspectos destacables y los puntos de mejora de los programas, es necesario someterlos a procesos de análisis constantes que permitan identificar datos, no únicamente a nivel cuantitativo sino desde la percepción de los actores a quienes está siendo dirigido el programa de manera que sea posible determinar las fortalezas y áreas de oportunidad.

Otro aspecto a destacar es la necesidad de contar con mecanismos que garanticen la confidencialidad de los datos y no se permitan cambios posteriores a las evaluaciones, de manera que se salvaguarde la veracidad de la información que se envía, puesto que son las situaciones como la que los profesores refirieron que sucedió en la escuela estudiada, sobre inventar información, que no permiten que exista la identificación de áreas de oportunidad que den lugar a las mejoras, adecuaciones y permitan la permanencia de programas implementados que, cuentan con amplias fortalezas en el ámbito educativo y socioemocional de los alumnos de educación básica.

Es fundamental la existencia y la propuesta de iniciativas federales como el PNCE que motiven, impulsen y orienten la creación de ambientes de convivencia en las escuelas, así como hacer visible la importancia de estos temas, los tiempos designados para este fin en los CTE no sólo deben permanecer, sino que también reforzarse y ampliarse. También, se debe mantener un seguimiento adecuado a los programas que permita la comunicación constante entre los miembros directivos del programa y las escuelas participantes encargadas de implementarlo puesto que, sólo de esa manera se puede vislumbrar los aspectos más destacables y las áreas de oportunidad. Es importante la educación continua a toda la comunidad educativa, especialmente en contextos donde prevalece la violencia. El PNCE debe ser evaluado por medio de instancias que permitan retroalimentaciones objetivas, confidenciales y seguras para los miembros de la coordinación general, quienes deben ser responsables de analizar e identificar las fortalezas y debilidades descritas por los participantes, todo siempre en la búsqueda de mantener y fomentar ambientes de convivencia pacífica que contribuyan a la educación en México. 


\section{LISTA DE REFERENCIAS}

Carro Olvera, A. (2018). Política educativa y convivencia escolar (1. ${ }^{a}$ ed.). México: gedisa.

Cerezo-Ramírez, F., y Sánchez-Lacasa, C. (2013). Eficacia del programa CIP para la mejora de la convivencia escolar y la prevención del bullying en alumnos de Educación Primaria. Apuntes de psicología, 31(2), 173-181.

Chaparro-Caso-López, A. A., Mora-Osuna, N., y Medrano-Gallegos, V. (2018). Estudio de la implementación del Programa Nacional de Convivencia Escolar (PNCE) en una entidad federativa mexicana. Psicoperspectivas, 18(1), 1-15.

Conde-Bonfil, C. (2017). Avances y retrocesos de la evaluación en México. Política y cultura, (47), 9-40.

Creswell, J. W. (2009). Research Design Qualitative, Quantitative, and Mixed Methods Approaches (3. ${ }^{\mathrm{a}}$ ed.). California: Sage.

Delors, J. (1996). Informe a la UNESCO de la Comisión Internacional sobre la educación para el siglo XXI, presidida por. MAdrid, España: Santillana.

Gobierno Estatal 2018-2024. (2018). Programa Nacional de Convivencia Escolar. SEGEY Secretaría de Educación. Recuperado de http://www.educacion.yucatan.gob.mx/programas/index

Martínez, A. M. M., y Góngora, D. P. (2011). Revisión sistemática de instrumentos utilizados para conocer los problemas de convivencia en los centros educativos. International Journal of Developmental and Educational Psychology, 3(1), 11.

Piña-Miramar, A. C., Tron-Álvarez, R., y Bravo-González, M. C. (2014). Acoso escolar en la educación secundaria: Percepción de los alumnos, profesorado y padres de familia. Revista Electrónica de Psicología Iztacala, 17(3), 1282-1307.

Rincón-Vargas, L. P. (2017). Evaluación del programa para la gestión del conflicto escolar Hermes en el Colegio Alfonso López Michelsen «IED»[Tesis de maestría]. Recuperado de https://repository.javeriana.edu.co/handle/10554/37889

Rivero Espinosa, E. (2017). La convivencia escolar: Perspectivas, conceptualizaciones y su gestión en Morelos. 12. San Luis Potosí, México.

Rossi, P. H., Lipsey, M. W., y Freeman, H. E. (2004). Evaluation: A systematic approach (7th ed). Thousand Oaks, CA: Sage. 
Saucedo Ramos, C. L., y Guzmán Gómez, C. (2018). La investigación sobre la violencia escolar en México: Tendencias, tensiones y desafíos. Cultura y Representaciones Sociales, 12(24), 213-245. https://doi.org/10.28965/2018-024-08

Secretaría de Educación Pública. (2016). Programa Nacional de Convivencia Escolar. Documento base. Subsecretaría de Educación Básica.

Secretaría de Educación Pública. (2017). Programa Nacional de Convivencia Escolar 2017- 2018. Consistencia y Resultados. Recuperado de Gobierno de México website: $\quad$ https://www.gob.mx/sep/documentos/programa-nacional-deconvivencia-escolar-2017-2018?state=published 